\title{
IMAGE SEGMENTATION METHODS FOR BRAIN MRI IMAGES
}

\author{
Swapnali Matkar ${ }^{1}$, Megha Borse ${ }^{2}$ \\ ${ }^{I}$ Dept. of Electronics \&Telecommunication, Cummins College of Engineering for women, Maharashtra, India \\ ${ }^{2}$ Assistant Professor, Dept. of Electronics \&Telecommunication, Cummins College of Engineering for Women, \\ Maharashtra, India
}

\begin{abstract}
In Image Processing, extracting the region of interest is a very challenging task. To extract information, pre-processing algorithms are important in MRI image. Edge detection is a task in which points in image are identified at which brightness changes sharply or it has discontinuities. It is an essential pre- processing step in medical image segmentation, for object recognition of the human organs. The applications of medical image segmentation are $3 D$ reconstruction and quantitative analysis and so on. We used MRI images because MRI images give best view of tissues in any part of human body. In this paper, difficulties of edge detection in brain magnetic resonance images are considered and a new approach to edge detection is introduced. There are many traditional edge detection methods for extracting edges from images have been introduced such as gradient based operators like sobel, prewitt, robert were initially used for edge detection, but they did not give sharp edges and were highly sensitive to noise image. And in medical field accuracy is important fact. To overcome these difficulties, we proposed new method called as Active Contour method or snake model. . In the field of medical segmentation, Active contour method is one of popular research topic. This method is used for detecting brain region based on their energy function. In order to compare between them, one slice of MRI image tested with these methods. The traditional and proposed edge detection algorithms are implemented in $M A T L A B$ and results of proposed method are presented and compared with traditional approach.
\end{abstract}

Keywords: Edge detection, Brain MRI images, Canny edge detector, Active contour method and MATLAB.

\section{INTRODUCTION}

Medical imaging is a significant application of image processing for clinical purposes such as diagnose, examine disease or for medical research. There are various types of imaging techniques are used to study brain anatomy and functions like CT scan, MRI, PET etc. But MRI images are widely used because they provide best visualization of abnormalities of brain also they provide high quality images with good soft tissue contrast.

The basic feature of image is Edge. In the image processing, edge detection is a very critical function since edge contains major information about image. The basic function of edge detection is to protect the important structural properties of image and filter out the useless information to reduce the amount of data. There are many edge detection methods exist for medical images. In this paper we make comparison between some of traditional methods and new approach for edge detection i.e. Active Contour Method.

In the first section we discussed different edge detection algorithms like robert, Prewitt, Sobel are Gradient based classical operators initially used for edge detection. These methods are used to find out approximate absolute gradient magnitude at each point in an input grayscale image but they did not give sharp edges and were highly sensitive to noise image. Canny edge detector uses Gaussian filter to denoised the image and then apply non-maximum suppression. In next section we discussed about the concept of active contours which was introduced by Kass. The two properties of Active contour i.e. smoothness and continuity, capture edge of curved object sharply. In the last section experimental results are shown and comparison is done between these methods.

\section{TRADITIONAL EDGE DETECTION METHODS}

We begin by introducing some of traditional approaches for edge detection.

1. Sobel Edge Detector

2. Prewitt Edge Detector

3. Canny Edge Detector

\subsection{Sobel Edge Detection [5]}

Sobel Edge detection method is very simple to implement. We have to compute the partial derivatives at every pixel location in the image to obtain the gradient of an image. These derivatives can be derived by using two $3 \times 3$ size masks. The mask is slide over image, one estimating the gradient in the $\mathrm{x}$-direction and the other estimating the gradient in the y-direction. 


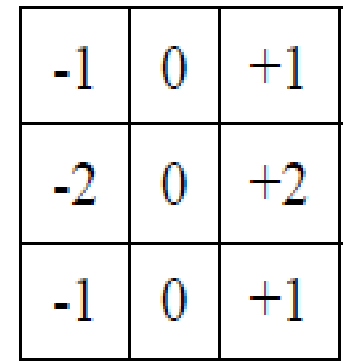

Gx

\begin{tabular}{|c|c|c|}
\hline+1 & +2 & +1 \\
\hline 0 & 0 & 0 \\
\hline-1 & -2 & -1 \\
\hline
\end{tabular}

\section{Gy}

Fig -1: Sobel convolution mask

We move the sobel mask over the MRI image to calculate horizontal and vertical gradient ( $\mathrm{Gx}$ and $\mathrm{Gy}$ ) ,then we combine it together to find magnitude and orientation of point. Magnitude gives us strength and orientation gives us direction of pixel

$$
\begin{aligned}
& \text { Magnitude }=\sqrt{ }\left(\mathrm{Gx}^{2}{ }_{+} \mathrm{Gy}^{2}\right) \\
& \text { Direction }=\tan ^{1}(\mathrm{~Gy} / \mathrm{Gx})
\end{aligned}
$$

\subsection{Prewitt Edge Detection [5]}

The implementation of prewitt edge detector is same as sobel edge detector but have mask of different coefficient. Both masks are convolved with the image and summed to get output.

\begin{tabular}{|c|c|c|}
\hline 1 & 1 & 1 \\
\hline 0 & 0 & 0 \\
\hline-1 & -1 & -1 \\
\hline
\end{tabular}

$\mathrm{G}_{\mathrm{X}}$

\begin{tabular}{|c|c|c|}
\hline-1 & 0 & 1 \\
\hline-1 & 0 & 1 \\
\hline-1 & 0 & 1 \\
\hline
\end{tabular}

$\mathrm{G}_{\mathrm{y}}$
Fig -2: Prewitt convolution mask

\subsection{Canny Edge Detection [1]}

Canny Edge detector is optimal edge detector. It works in multi-stage process. The algorithm of canny detector has following steps:

1. Smoothing using Gaussian Filter

2. Calculate gradient magnitude

3. Non maxima suppression

4. Thresholding

In order to remove the noise, filter the image is very essential step. Gaussian filter is convolved with image to obtain smooth image. Then by using sobel or prewitt operator, we find gradient magnitude and orientation. After calculating gradient magnitude we get blurred edges in the image. To convert the blurred edges into sharp edges we have done non maxima suppression. In non- maxima suppression, edge strength of current pixel is compare with pixel in +ve and -ve direction. If current pixel magnitude is larger than other pixels, keep the value of the magnitude otherwise suppress the value. To detect and link the edges, we use double threshold algorithm. If we use only low threshold, noisy maxima will get added and if we use high threshold, there is a possibility of true maxima might be missed. Therefore in canny method double threshold is used. The strong edge points than high threshold are set as strong and points weaker than low threshold are suppressed and points between thresholds are set as weak.

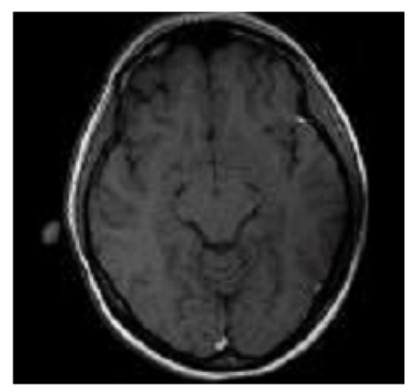

Original Image

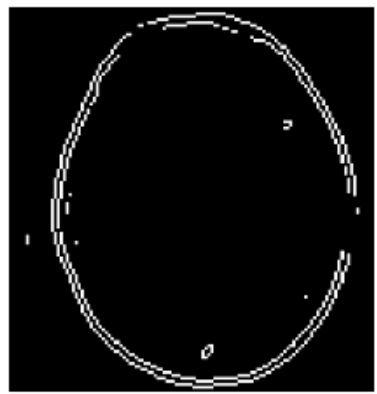

(2) Prewitt

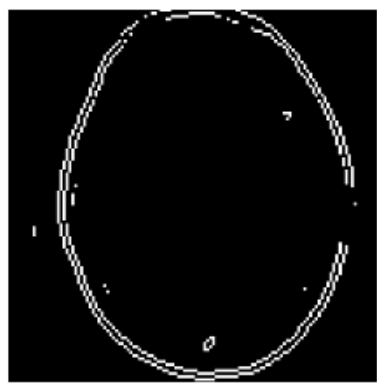

(1) Sobel

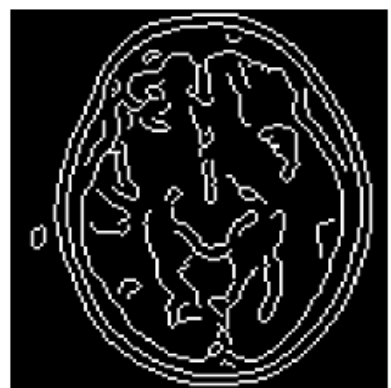

(3) Canny
Fig -3: Comparision between sobel,prewitt and canny detector

\section{PROPOSED EDGE DETECTION METHOD [2]}

The difference in grey level values in the medical images are not very clear, therefore the edges of organs are always vague. Often edge obtain after canny detector is not continuous, to improve segmentation, Active contour method have been widely used in medical image analysis. It is also called as snake model. The snake model depends on the energy function of curve. Snake model is a parametric curve which is under influence of internal and external energies. Where energy function of edge is minimum, curve tries to converge in that direction.

Basic Principle:

The basic contour curve is defined as:

$$
\mathrm{V}(\mathrm{s})=(\mathrm{x}(\mathrm{s}) \cdot \mathrm{y}(\mathrm{s}))
$$

Energy function is given as :

$$
\text { E_model=E_int }+E_{-} \text {ext(includesE_img) }
$$


There are basically three energies functions:

1. Internal energy function

2. External energy function

3. Image energy function

By using $E_{\text {int, }}$ the internal energy we can control the shape and smoothness of contour. $\mathrm{E}_{\text {int }}$ can be written as:

$$
\mathrm{E}_{-} \text {int }=1 / 2\left[\int \alpha\left|\mathrm{v}^{\prime}(\mathrm{s})\right|^{2}+\beta\left|\mathrm{v}^{\prime \prime}(\mathrm{s})\right|^{2}\right] \mathrm{ds}
$$

Internal energy gives smoothness and curvature. In the above equation, the measure of ealsticity has been given by first order term of contour curve. The $2^{\text {nd }}$ oeder term gives measure of curvature. The elasticity value is high where curve get streched and the curvature value is high where curve get kinked. $A$ and $\beta$ are continuity and degree of bending paraments respectivily. We can get more deformation by lowering these parameters.

$E_{\text {ext, }}$, the external energy itself includes the image energy. The external energy helps to guide the behaviour of segmentation. And the image energy helps for durecting the deformation of contour, It pushes the contour model towards the position of object edge. The Steps for Active contour model are given as follows: [3]

1. First we set the initial contour around the object boundary. This initial contour should be close to the boundary to get the close deformation.

2. Plant the searching lines. And on each searching create candidate points.

3. Calculate the energy for each candidate point using energy function formula.

4. Select the suitable points with minimum energy. Minimum energy shows better possibilities to be close to object contour.

5. Connect that best matched points to form closed contour. From above procedure segmentation is done completely.

\section{EXPERIMENT}

To perform different edge detection methods we used brain MRI image of type T1 weighted. The size of image is $131 \times 131$. First convert the image to grayscale image before applying the edge detection algorithm. All the operations are performed in MATLAB. The output of sobel, prewitt and canny edge detector, we can see in figure 3 . The sobel and prewitt operator did not show detail information because most of the data is lost. For the further study in medical image analysis required detail information which cannot produce by using these edge detector. Canny edge detector can produce output with better localization. Also it is less sensitive to noise unlike sobel and prewitt edge detector. It works fine under the noisy condition. But if we set threshold value too high, then we can loss some important information. And if threshold value is too low, then some unwanted information might be get added. So giving threshold value which works well on all images is very difficult.
Active contour model has interactive ability and overcomes shortcomings of traditional methods. This method gives better output, once an appropriate initialization is done. The brain image is more concave. By using this method this concave region can be processed in better way. [4]

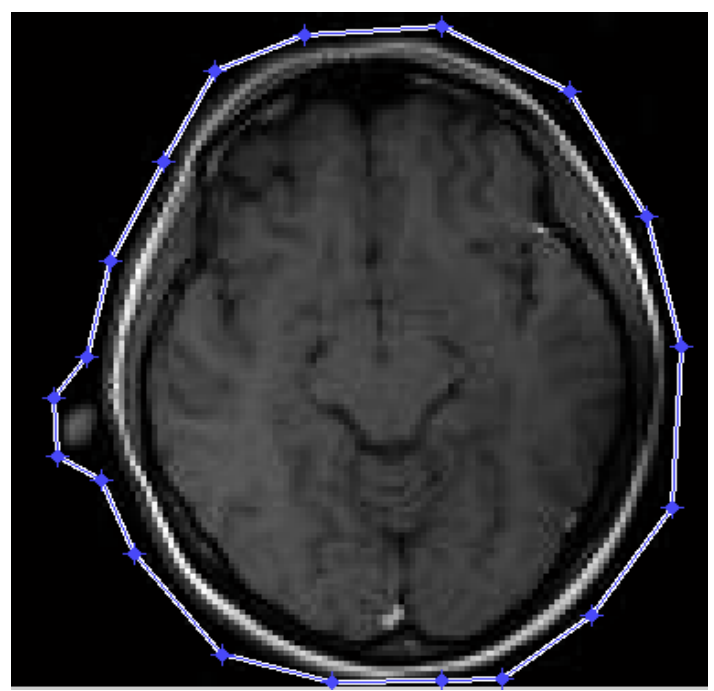

Fig -4: Initial contour around object boundary

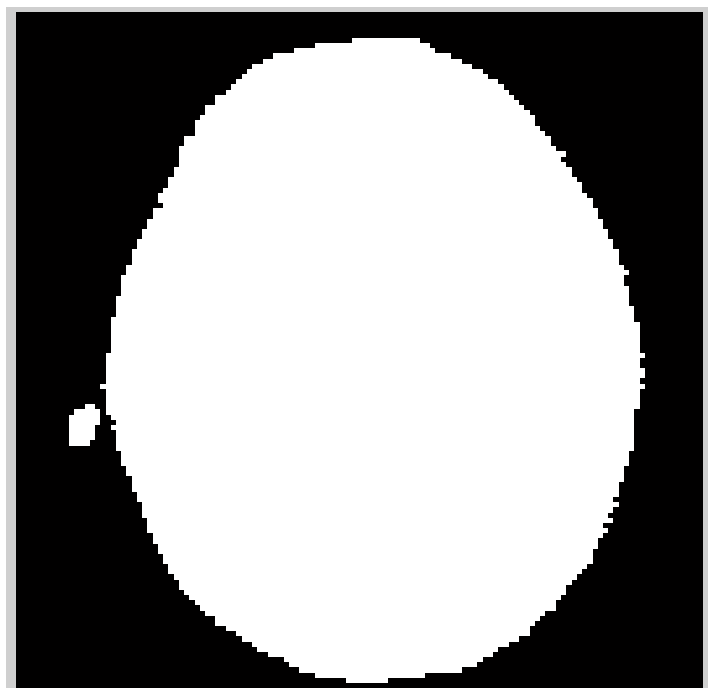

Fig -5: Final Segmented Imag

These traditional methods were compared with active contour method using two different criteria.

1) Visual quality comparison 2) PSNR value.

A larger PSNR value indicates better quality of image. After analyzing the PSNR of the test cases, it is clear that the active contour method performed well on the image.

Table 1 compares PSNR values of these methods.

\begin{tabular}{|l|l|}
\hline Edge Detection Method & PSNR Value \\
\hline Sobel Edge Detection & 13.9112 \\
\hline Prewitt Edge Detection & 13.9107 \\
\hline Canny Edge Detection & 13.9222 \\
\hline Active Contour & 43.2072 \\
\hline
\end{tabular}




\section{CONCLUSION}

In the medical image processing accuracy is the important factor. The output of sobel and prewitt edge detector is not enough accurate with the thin and smooth edges. In canny edge detector, sometimes edge is spurious and they contain holes along curve which is not desirable. Also in the traditional methods, we convolve image with horizontal and vertical gradient operators which gives detection of edge in favour of rectilinear object only. In the field of medical segmentation, Active contour method is one of popular research topic. Two important properties of Active contour such as smoothness and continuity increase the accuracy of segmentation. It is more suitable to capture curved object boundaries. In comparison with traditional method of edge detection, Active contour method gives better segmentation and accuracy.

\section{REFERENCES}

[1]. Zolqernine Othman, Habibollah Haron, Mohammed Rafiq Abdul Kadir, "Comparison of Canny and Sobel Edge Detection in MRI Images", University Teknologi Malaysia,Pg.no. 133-134.

[2]. Jinyong Cheng, Yihui Liu, Ruixiang Jia, Weiyu Guo, "A New Active Contour Model for Medical Image Analysis Wavelet Vector Flow", IAENG International Journal of Applied Mathematics, Volume 36, Pg.no. ,429-430,May 2007.

[3]. Jinyong Cheng, Yihui Liu, "3-D Reconstruction of Medical Image Using Wavelet Transform and Snake Model", JOURNAL OF MULTIMEDIA, VOL. 4,Pg.no428-430,(2009).

[4]. Jianhua Liu, Jianwei Wang, "Application of Snake Model in Medical Image Segmentation", Journal of Convergence Information Technology(JCIT), Vol.

9,Pg.no-105-106,2004.

[5]. R.C.Gonzalez and R.E.Woods, "Digital Image Processing",Addison-Wesley,Reading,Pg.no.744 ,(1992).

\section{BIOGRAPHIES}

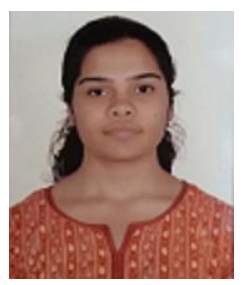

Ms.Swapnali Matkar is pursuing M. E. degree in Signal Processing from Cummins College of Engineering for women under Pune University. She is received B.E degree in Instrumentation from Mumbai University

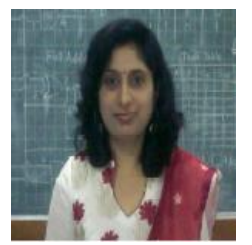

Mrs..Megha Sunil Borse received M.E.degree in Electronics Digital Systems from Pune University. She is registered $\mathrm{PhD}$ student of Pacific Uniersity, Udaipur. She is currently working as a Assistant Professor in Cummins College of Engineering for women, Pune. She has total 16 years of teaching experience. Her area of specialization is biomedical image processing. 